\title{
Digital Shape and Geometric Dimension Analysis of Polymer Fuel Tanks
}

\author{
Jerzy Józwik ${ }^{1}$, Krzysztof Dziedzic²* \\ 1 Department of Production Engineering, Mechanical Engineering Faculty, Lublin University of Technology, \\ Nadbystrzycka 36, 20-618 Lublin, Poland \\ 2 Institute of Computer Science, Electrical Engineering and Computer Science Faculty, Lublin University of \\ Technology, Nadbystrzycka 38, 20-618 Lublin, Poland \\ * Corresponding author's e-mail: k.dziedzic@pollub.pl
}

\begin{abstract}
3D optical scanning method, is employed to digital shape and geometric dimension analysis of fuel tanks. The following paper presents the application possibilities of the optical 3D measurement method for measuring and archiving dimensions of geometrically complex parts, on the example of a passenger car fuel tank in local company. Based on the conducted measurements, for a specific type and size of the population of fuel tanks for vehicle, authors proposed model that classifies the geometric accuracy of the tanks produced in the production line. For the analysis and data processing different informatics techniques have been used. The main objective of theirs was to identify the possibility of implementing the system for automation of the measurement to the production line. The measurements were implementation at local company - a manufacturer of polymer tanks in the automotive industry. For most of the measuring points, the mean value of the measuring deviations ranged from $-1 \mathrm{~mm}$ to +1 $\mathrm{mm}$. The mean values of the standard deviations are presented for a representative deviation of the $\Delta \mathrm{X}$ dimension of $0.1 ; 5 ; 10 ; 15$ and $20 \mathrm{~mm}$ were respectively: $0.014 ; 0.150 ; 0.172 ; 0.289 ; 0.340 \mathrm{~mm}$. The dimensional shape assessment of the manufactured tanks is extremely important from the point of view of the automation of this task in the production line. The optimization of the measurement of tanks carried out in laboratory conditions was implemented on the production line. The main task of optical scanning for the assessment of dimensional conformity has not been used so far in factory. This is due not only to the cost of such systems, but also to certain limitations that have been resolved thanks to laboratory tests. The obtained results of the metrological inspection make it possible to identify significant dimensional and shape deviations of automatically manufactured tanks. The proposed use of artificial neural networks allows the production with a high quality of tanks accuracy with the minimum the number of scanned tanks in the production line. The paper also presents the possibilities of the computer software to analyze the deviations of geometrically very complex parts.
\end{abstract}

Keywords: fuel tanks, reverse engineering, dimensions measurement, 3D scanner.

\section{INTRODUCTION}

The use of products made of polymers and polymer composites is very wide. Parts or components made of polymeric materials are most often found in the automotive, aviation, medicine and a composite pair friction materials (bearing guides). Physical and chemical properties of polymeric and polymer composite materials were analysed by many authors in their papers. On paper [1] authors' analysis tribological properties (friction coefficients and temperature near nodes) of selected polymer composites using especially for tribological pairs (bearings). In the article [2] an analysis of friction and wear behaviors of everal polymers sliding against $\mathrm{GCr} 15$ and 316 steel under the lubrication of sea water was presented. The direct-friction riveting of polymer composite laminates for aircraft applications was analysed in [3] but on paper [4] and [5] the authors' analysis bending behaviour of polymeric materials used in biomechanics orthodontic appliances and wear resistance with borided C45 steel contact. Literature analysis shows that polymer plastics and 
composite materials can successfully compete with metal alloys in terms of strength properties [6]. In addition to very good mechanical properties, polymer composites are characterized by high corrosion resistance, ease of moulding, absorption of mechanical vibrations, as well as the absence of electrical conductivity and low thermal conductivity and good tribological properties $[7,8]$. In the automotive industry, polymer materials are used to produce such elements as: dashboards, fuel tanks, strips, bumpers, wires, rims, hubcaps, engine covers, spoilers, fairings, pedal covers, license plate frames, engine timing covers, mirror housings, boot covers, battery housings, carpets, bodies, air inlet grilles, fenders and parts of the outer sheathing [9]. Optical digitization is used in a wide variety of applications. The specific applications of the measurement process using 3D measurement systems are: light structure systems, wind turbine, cutting parts, analysis of tool wear, additive manufacturing (AM) and $\mathrm{CNC}$ machine tools evaluation, forging defects, layer inspection of ceramic materials after milling and another like: welding, verification of ram-press pipe, digitizing press parts and tools or analysis of relation between the 3D printer laser beam power and the surface morphology [10-13].

One of the most important and responsible applications of polymers in the automotive industry is the production of fuel tanks, which until recently were manufactured as metal tanks. In the 1970s, it turned out that the cars of one of the well-known manufacturers had a fatal design flaw due to a poorly designed and improperly positioned fuel tank, which tended to explode in rear-end collisions at speeds exceeding $32 \mathrm{~km} / \mathrm{h}$. Thus, it turned out that the material and construction of the tank have a significant impact on the safety of passengers. This is what forced the use of materials and technologies that would ensure that the tanks are safe in usage. This is ensured by the use of polymeric tanks. The use of tanks made of macromolecular materials makes their structure light and reduces the weight of the vehicle, and thus fuel consumption, which gives measurable financial benefits. Today, most fuel tanks today are polymer tanks. Car fuel tanks are geometrically complex products, produced as multi-layer structures, especially responsible for car safety [14]. The construction of the tank is typically multi-layered and requires extrusion blow moulding. This process should be fully controlled. In order to maintain the highest manufacturing standards, tanks should be subjected to a scrupulous inspection process and their quality monitored. This happens in production lines and at the final stage of quality control [15]. Measurements and assessment of the geometrical compatibility of the tank with the model can be performed using tactile or non-contact methods. Tactile methods are based on measuring machines and mobile systems, commonly used to measure metal parts in the machinery sector [16]. Touch methods are time consuming and require qualified personnel to handle them. An alternative to tactile methods of measuring and monitoring the dimensions of fuel tanks is the use of optical 3D measuring techniques. In [17, 18], 3D scanning was used in relation to the $3 \mathrm{D}$ printed parts. High data quality, a large number of measuring points - giving information on the entire surface of the measured product, and direct comparison with CAD data are the main advantages when choosing an optical measuring method. Real-time 3D shape inspection system of automotive parts based on structured light pattern was presented in work [19]. 3D Recognition of car parts in automotive supply chains by combining synthetically generated training data with classical and deep learning based image processing is presented in [20]. Authors [22, 23] an application of the 3D reverse scanning method used for the analysis of tool wear and forging defects. Authors of paper [24] presents design, manufacturing and test of CFRP front hood concepts for a light-weight vehicle. The manufacturing steps and full details regarding the fabrication process are presented and $3 \mathrm{D}$ scanning techniques were used to reveal the manufacturing high dimensional accuracy with respect to initial CAD models. In another paper [25] authors prefer novel method for the manufacture of complex CFRP parts using FDM-based molds. This paper features a new design of bike saddle, which was manufactured through the use of molds created by fused deposition modeling (FDM), of which two 3D printable materials were selected, polylactic acid (PLA) and acrylonitrile butadiene styrene (ABS), and these molds were then chemically and thermally treated. Following manufacture the molded parts were subjected to a quality inspection, using non-contact three-dimensional (3D) scanning techniques, where the results were then statistically analysed. Authors of papers [26, 27] present methodology for evaluating the form deviations for formula one nose car methods for checking the symmetry of the formula one car nose. In these papers authors also use (3D) laser 
scanning techniques for measurement evaluation. Laser scanning the amount of acquired points increases considerably and facilitates a more exact determination of a trend. The authors use laser scanning process on CMM measurement machine. The literature analysis carried out showed that, the optical measurement method is used to dimension objects with complex geometry - such as fuel tanks, polymer and polymer composite parts, where other techniques do not work or their use is complicated, time-consuming and problematic.

\section{MATERIALS AND METHODS}

During spatial scanning, blue light is emitted towards the examined object through an interference mesh with a structure ensuring obtaining moire fringes. Such a system of fringes refracts on the measured surfaces, and partially reflected is sent as a new system to the image analyzer through the CCD matrix. For the process to run properly, the intensity of the fringes must be adjusted to the requirements of the phase shift. During the tests, the ATOS Compact Scan measuring system uses the optical triangulation method [23]. The optical triangulation method is one of the most popular 3D methods of acquiring data about the measured object. The theoretical description of this method was given in 1615 by W. Snell van Raven. This method is one of the extremely precise measurement methods. Triangulation involves measuring the angles between all adjacent points and the length of at least one side in a network of triangles. In the optical triangulation method, a laser beam (in the pulse mode) is directed at the measured object, the beam of which, after reflecting from the surface of the measured object, goes to the receiver, usually a CCD detector - a system of many photosensitive elements, each of which records
Table 1. Parameters of the ATOS Compact Scan 3D scanner

\begin{tabular}{|l|c|}
\hline \multicolumn{1}{|c|}{ Parameter } & ATOS Compact Scan 2M \\
\hline Camera Pixels & $2 \times 2000000$ \\
\hline Measuring Area & $35 \times 30-1000 \times 750 \mathrm{~mm}^{2}$ \\
\hline Point Spacing & $0.021-0.615 \mathrm{~mm}$ \\
\hline Working Distance & $450-1200 \mathrm{~mm}$ \\
\hline Sensor Dimensions & $340 \mathrm{~mm} \times 130 \mathrm{~mm} \times 230 \mathrm{~mm}$ \\
\hline Operating Temperature & $5-40^{\circ} \mathrm{C}$, non condensing \\
\hline Power Supply & $90-230 \mathrm{~V} \mathrm{AC}$ \\
\hline
\end{tabular}

and then allows to read an electrical signal proportional to the amount falling on light it. The receiver is at a fixed distance from the transmitter, known as the base, and is tilted at an angle $\alpha$. A light beam reflected from an object with a known distance falls on the CCD detector behind the receiver lens. The change in voltage across the CCD detector is directly proportional to the known distance. Knowing the focal length of the lens and the place where the light beam hits the detector, we can determine the distance from the object. The TRITOP system - Optical 3D Coordinate Measuring Machine and the ATOS Compact Scan 3D scanner manufactured by GOM (with the parameters shown in Table 1.) were used.

The ATOS Compact Scan non-contact measuring system is used to perform quick and precise measurements of the geometry of objects. Due to its accuracy and versatility, this system is used on a large scale in industry and research centers.

The measurement process and the possibility of data analysis were carried out on the example of a passenger car fuel tank, the original and geometric model of which is presented in Figure 1.

The ATOS Compact Scan measuring system uses a special method of measuring and projecting developed by Gesellschaft für Optische Messtechnik. Thanks to the use of this innovative technology, it is possible to precisely scan objects with complex shapes. Using blue light

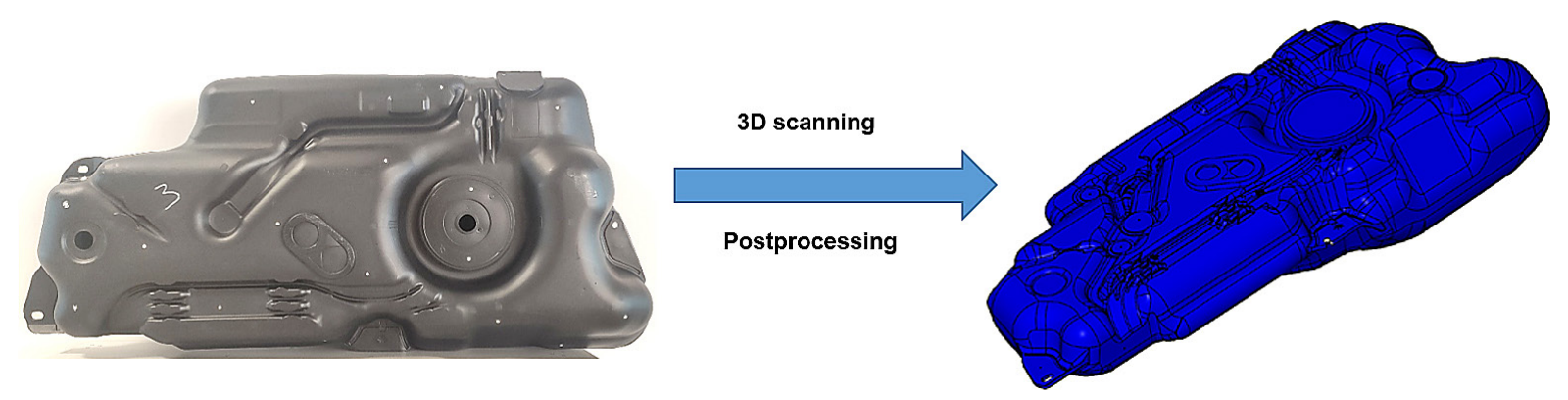

Fig. 1. Fuel tank a) original, b) geometric 3D model 
technology, the surface scanning process has become independent of external lighting conditions. Before starting the scanning process, it is important to properly prepare the elements to be scanned. The test object should be glued with reference points. Their task is to provide relevant information about the entire scanned object, mainly about overlapping areas (when combining subsequent scans), but also about its location and size.

In the measurement process, two CCD cameras were used, which provide several million measurement points within a few seconds. The head projector projects a sequence of fringes on the measured object, and the two CCD cameras record their course. By solving the optical transformation equations, the system calculates the coordinates for each camera pixel with high accuracy. The result of a single measurement is a cloud of points or a triangle mesh that uniquely reflects the scanned surface.

Figure 2 presents the projection of the sequence of fringes on the measured object. The problems that arise during scanning is the reflective surfaces and the uneven lighting that changes the image of the fringes. Both limitations reduce the accuracy of the measurement, although they can be eliminated, or at least their negative effect can be reduced by using blue light in the projector.

The GOM Atos software, in which the scanner operates, has many useful functions. This software controls the condition of sensors and the process of creating a point cloud on an ongoing basis during the measurement.

With the use of this software, we can edit the created point cloud on a current basis, thanks to which the measurement results can be better used in the fields of quality control, production process and reverse engineering. The aforementioned software has many certificates confirming its high accuracy in measurement processes.

It should also be noted that during the measurements the ATOS system constantly records, shows and saves measurement photos along with all relevant information about the quality of the measurement

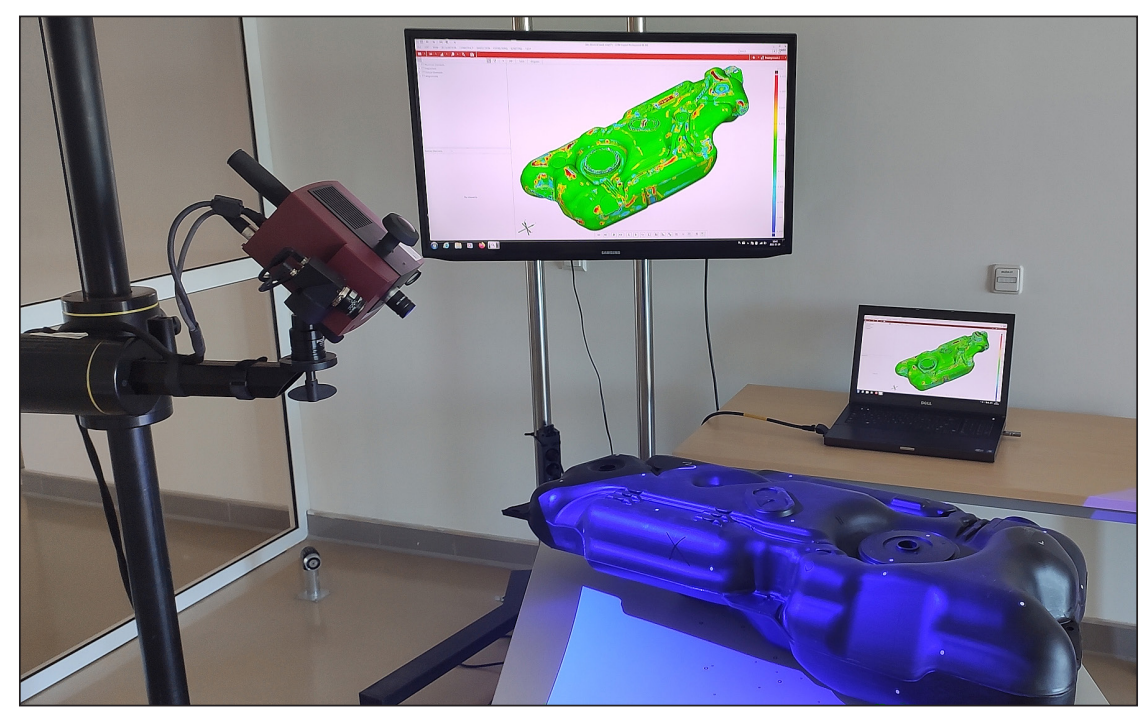

Fig. 2. Projection of the sequence of fringes on the measured object

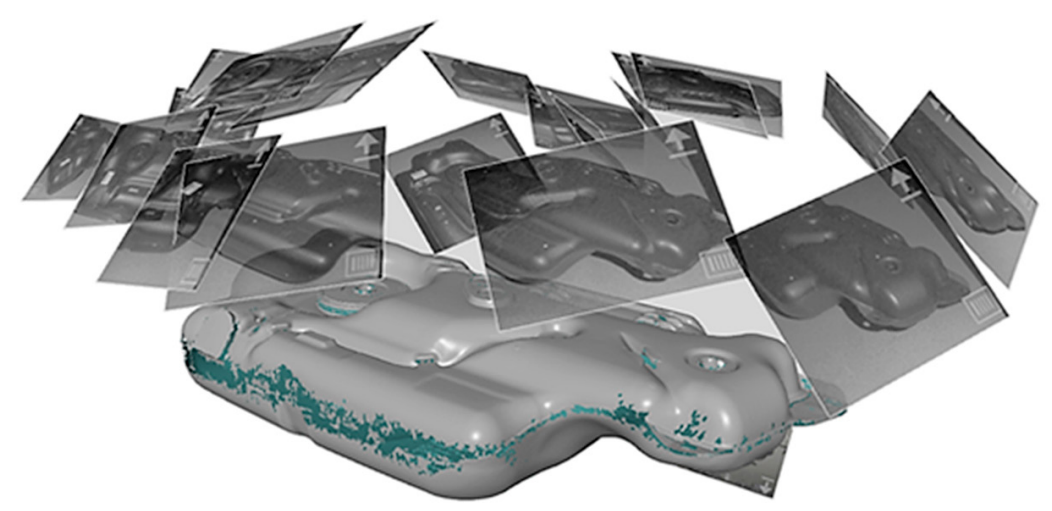

Fig. 3. Projection of the sequence of fringes on the measured object 
throughout the scanning process, thanks to which it is possible to recreate the conditions in which the measurement was carried out (Fig. 3).

\section{RESULT AND DISCUSSION}

\section{Quality control of passenger car fuel tanks}

The result of measuring the fuel tank of a passenger car with the ATOS scanner is an optimized triangle mesh that contains information about the entire surface of the scanned matrix (Fig. 4).

After scanning, the data obtained must be properly based and exported in *.STL format. The CAD data is then imported into the ATOS software, where the measured part is based to obtain the best fit. The result of comparing the measurement data with the CAD data is a clear and easy to interpret coloured map of deviations. Cold colours mean that there is a loss of material in relation to the CAD model, warm colours are excess material. Additionally, the map can be enriched with inspection points attached in any place, which inform about the numerical value of the deviation. It is also possible to dimension the most important features of the forging according to a 2D drawing (angles, distances, hole spacings, etc.). The result of the analysis can be a professionally prepared measurement report, which can be exported to popular formats (*.pdf, HTML, *.doc, etc.). Thanks to the free GOM Inspect software, it is possible to view and analyse the scan results on multiple computers at once. Another interesting application of 3D scanning is the archiving of fuel tank measurement data. It very often happens that successive container moldings differ slightly from each other, which is the result of a complex spectrum of interactions.

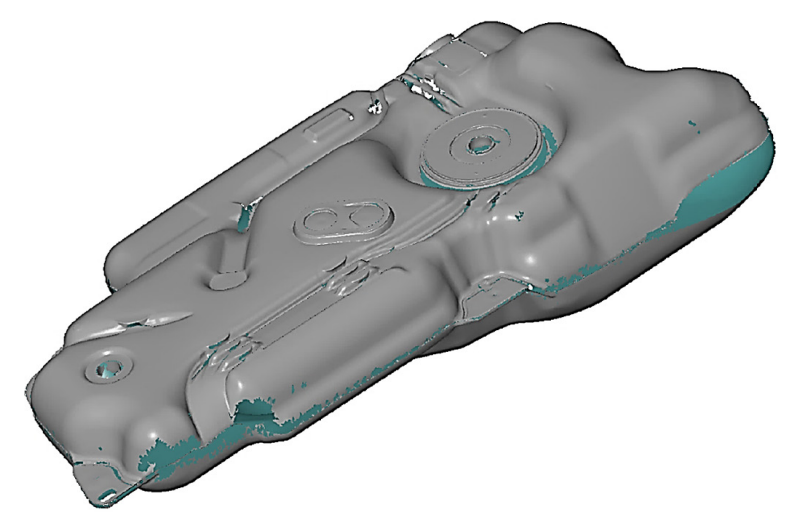

Fig. 4. The scanned model of the fuel tank in the form of a triangle mesh
Building a knowledge base about the geometry of the reservoir will allow the construction of appropriate models and forecasting the geometry of subsequent manufactured tanks. The introduction of fuel tanks to the production line will allow to minimize the time wasted in preparing the measurement and writing measurement programs.

\section{Analysis of deviation maps and constructing a prognostic model}

The basic form of presenting the results is a coloured map of deviations. It can only be obtained when a CAD model is delivered to the scanned element. If this condition is met, we can obtain a coloured visualization of the deviation maps as shown in Figure 5 by superimposing the CAD model on the grid of STL points.

This way of presentation allows us to quickly assess the differences between the ideal model created by the designer during the design of the CAD part and the actual product obtained in the moulding process. This method of showing the results is not very accurate, even despite the scale provided in the deviation map, therefore, after such a preliminary analysis, detailed inspection sites or sections are created in which the discrepancy is examined (Figs. 6-7).

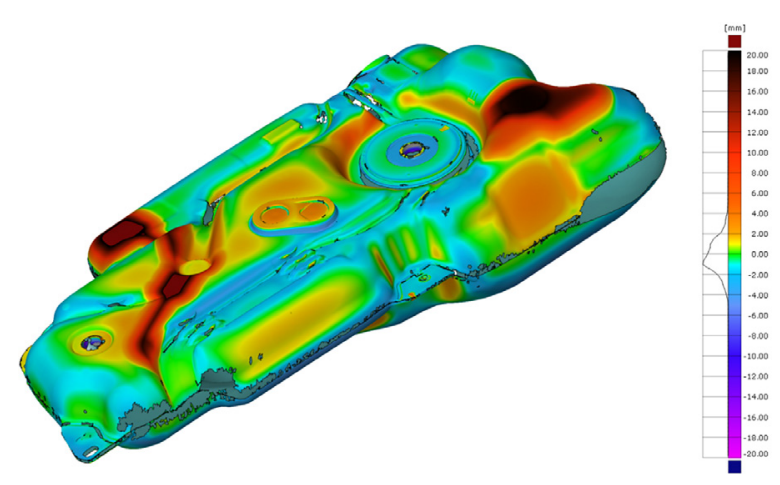

Fig. 5. View of the coloured map of deviations

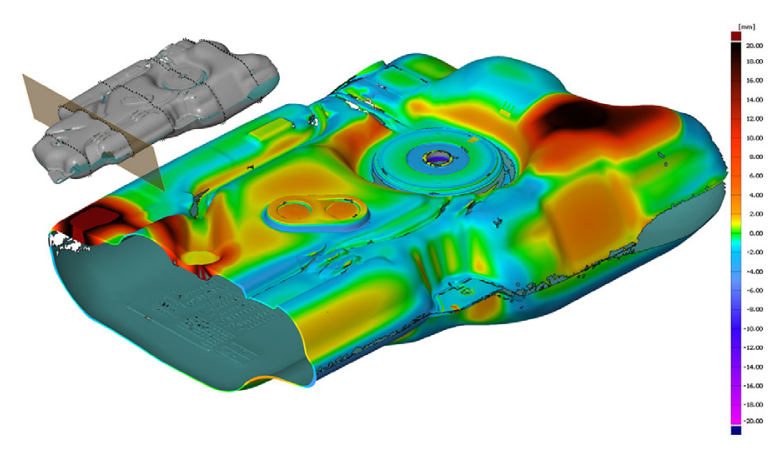

Fig. 6. Cross-section at the site of the largest anomaly 


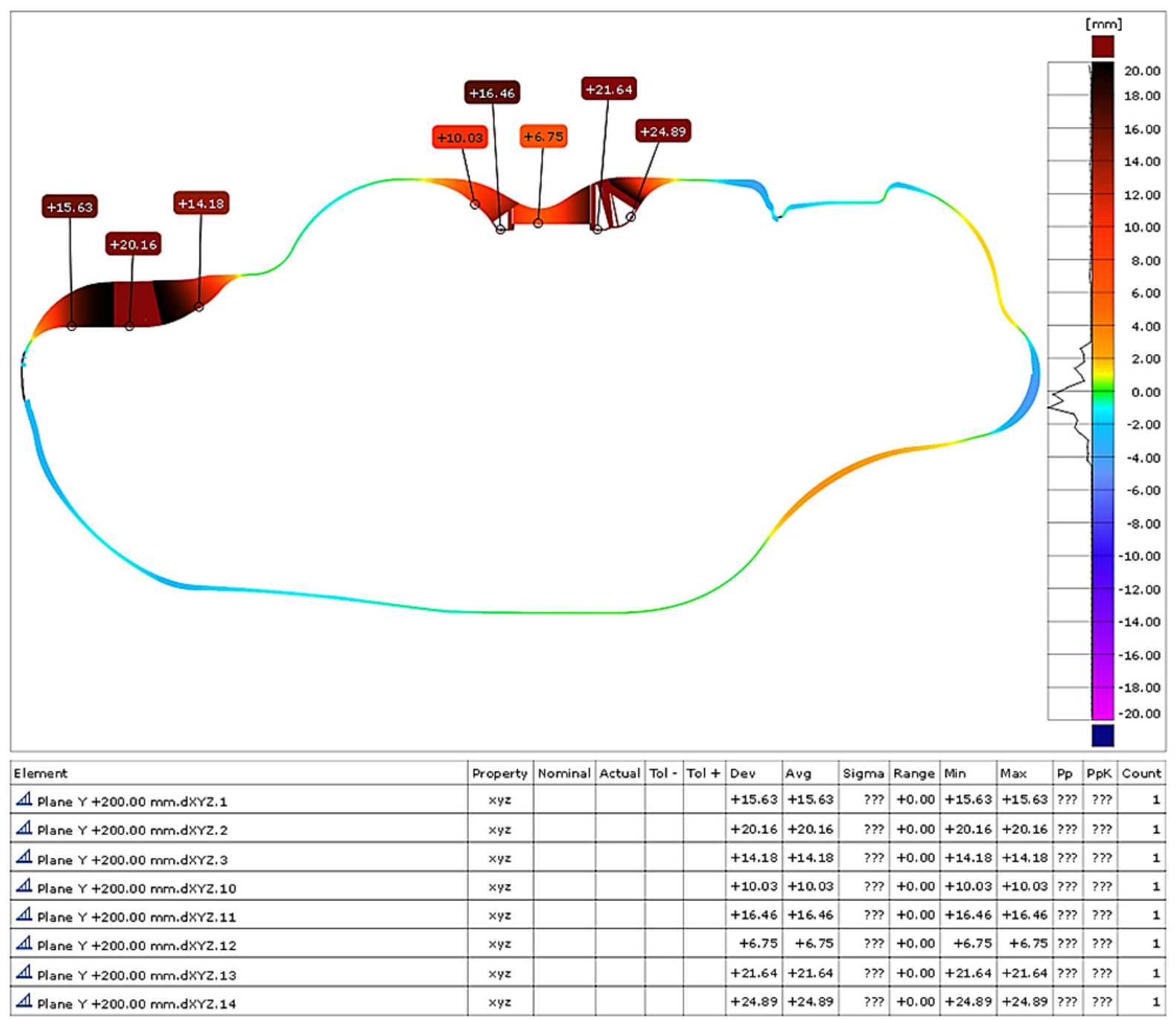

Fig. 7. A view of the deviation map of a given section with points of deviation

After selecting the appropriate section, we can introduce characteristic points from which we can read the direction and value of the deviation in relation to the model with nominal values. Figure 8 shows the cross- -section of the model for which the measurement points were discretized, for which deviations from the nominal dimension of the model were determined. You can specify any number of measuring points. A greater number of measurement points allows to precisely determine the deviations of the crosssection under analysis. However, not always a large number of measurement points is necessary. It depends on the expected metrological end result, the dimensions of the object and the required accuracy of measuring deviations in its dimensions. The accuracy of determining dimensional deviations depends on the accuracy of manufacturing the analysed part. Increasing the number of measuring points extends the time of the dimensional-shape analysis process.
Figure 9 shows the measurement planes for which a detailed quantitative analysis of deviations is possible. It shows also the generated results of the deviation analysis for the cross-section described in Figure 8 (number 4).

Figure 10 shows the average values of the standard deviation $s$ for 5 randomly selected tanks from the production line, for the deviations from cross-section number 4 . The mean values of the standard deviation are presented for a representative deviation of the $\Delta \mathrm{X}$ dimension of $0.1 ; 5 ; 10$; 15 and $20 \mathrm{~mm}$. From the presented representative calculation results, it can be concluded that the value of the standard deviation can be described by a linear function. Due to significant discrepancies in the value of dimensional deviations in the selected batch of fuel tanks in the analyzed cross-section no. 4 (analyzed due to significant dimensional errors), the values of the standard deviation are as high as $0.340 \mathrm{~mm}$. The minimum value of the standard deviation was identified for a dimension 


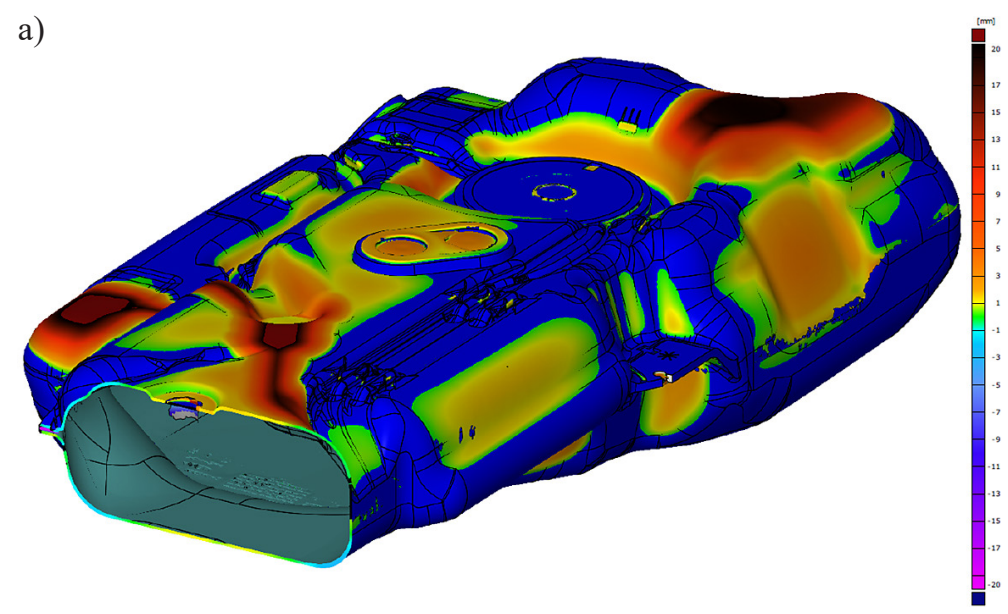

b)

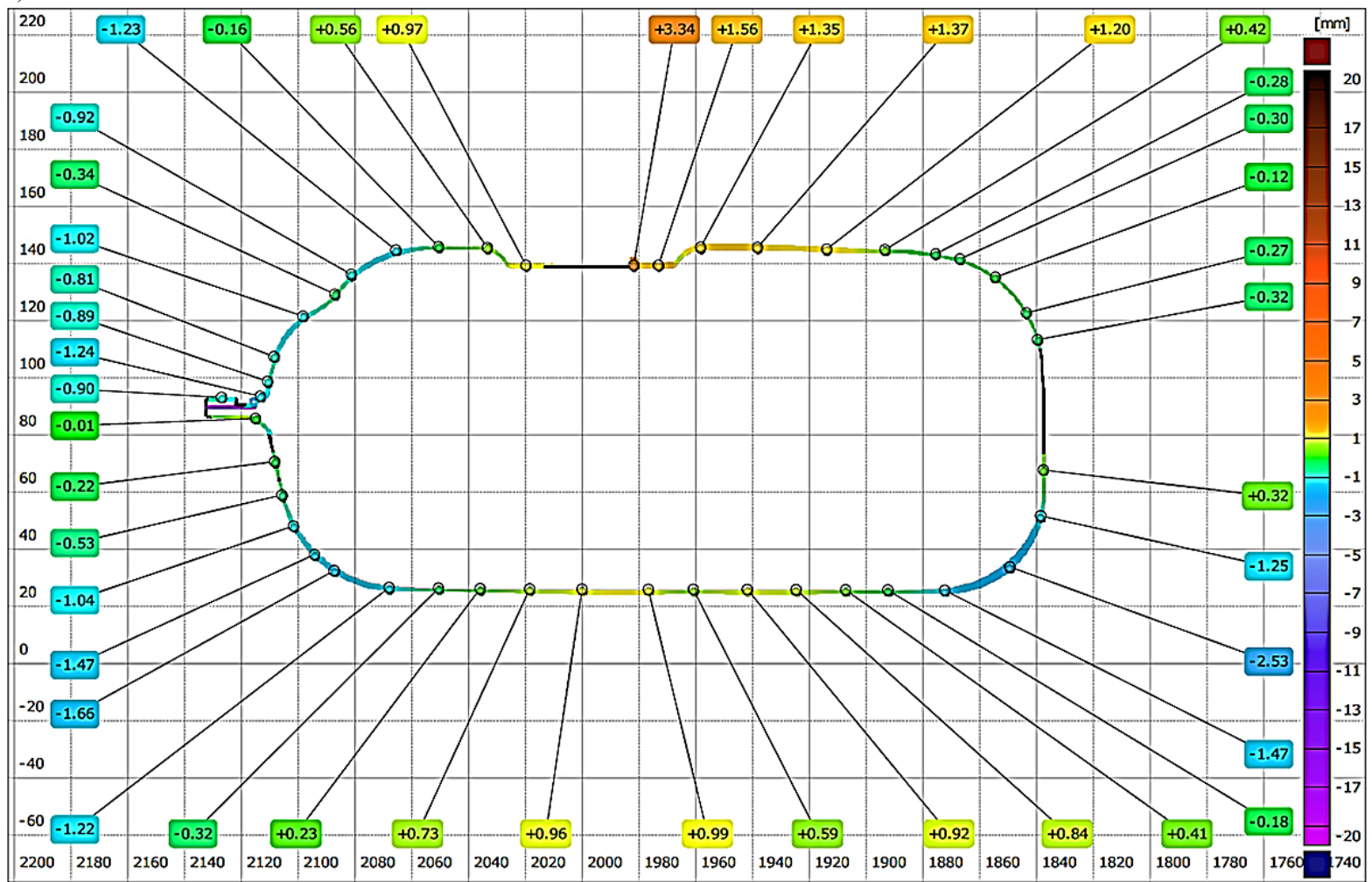

Fig. 8. Example of a dimensional analysis: a) cross-section on the model, b) discretization of the measurement points for which deviations from the nominal dimension have been determined

deviation of 0.1 and was respectively $\mathrm{s}=0.014$ $\mathrm{mm}$. The value of the minimum mean standard deviation roughly corresponds to the measuring accuracy of the ATOS measuring system.

Based on the conducted measurements, for a specific type and size of the population of fuel tanks, it is possible to build an intelligent model that classifies the geometric accuracy of the tanks produced in the production line (Fig. 11). The quality of the reservoir would be assessed on the basis of the current geometric information and compared with the patterns stored in an intelligent database. Such an approach would allow, according to strictly defined criteria, to classify a given tank into one of two groups: products that are geometrically appropriate and a group of tanks unacceptable from the point of view of dimensional and shape deviations. The constructed system would allow properly constructed tanks for operation, while the tanks with errors would qualify for the recycling process, allowing the recovery of the raw materials used.

The classifier presented in Figure 11 together with the automated measuring system, thanks to its integration into the production line (e.g. in the form of a scan box), would allow to create a maintenance-free decision-making system, bringing measurable utilitarian benefits. 

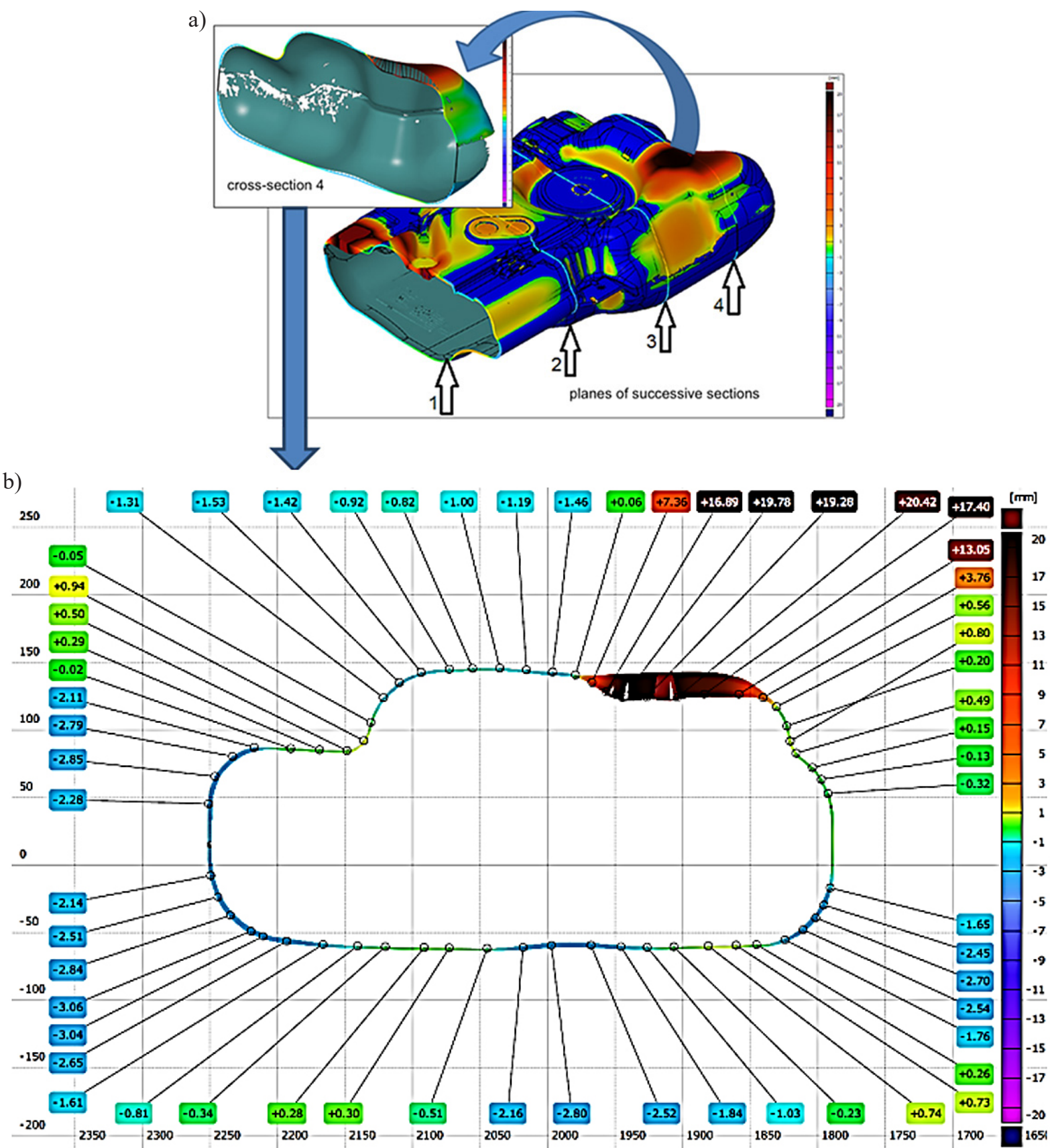

Fig. 9. Discretization of the scanned fuel tank by cross-section planes $(1,2,3,4)$

The use of the Atos system in the intelligent structure of the accuracy classifier for manufacturing fuel tanks (Fig. 12) would allow to shorten the production process, increase the number of geometrically controlled tanks, and increase the reliability of the measurement system. The use of non-contact measurements would also eliminate many measurement errors associated with tactile measurements.

\section{CONCLUSIONS}

As a result of the measurement process of the passenger car fuel tank geometry, a 3D virtual model was obtained, which was the basis for metrological analyses. The conducted tests showed significant dimensional and shape discrepancies the tested tanks. The values of the identified dimensional deviations at the measurement points indicated in the experiment are in the maximum range from $-25 \mathrm{~mm}$ to $+25 \mathrm{~mm}$ in relation to the nominal size of the tested tanks. The high value of dimensional deviations identified at the same points of all tested fuel tanks is caused by defects in the manufacturing process or the construction of the tool itself (blow mold). However, for most of the measuring points, the 


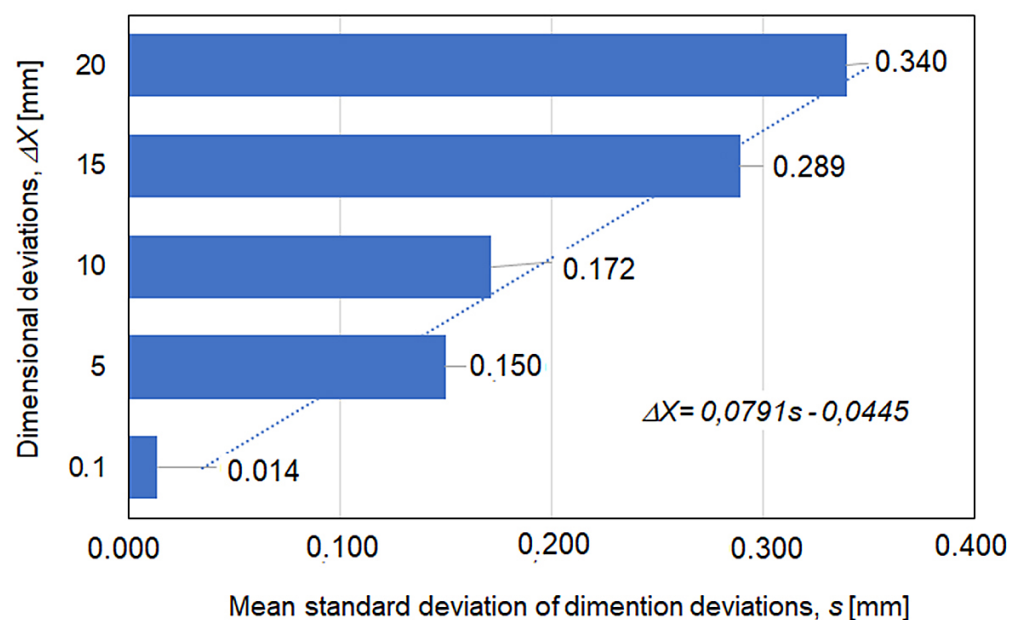

Fig. 10. Average values of the standard deviation for 5 randomly selected tanks from the production line, for the deviations from cross-section number 4 (for the measured deviations $0.1 ; 5 ; 10 ; 15$ and $20 \mathrm{~mm}$ )

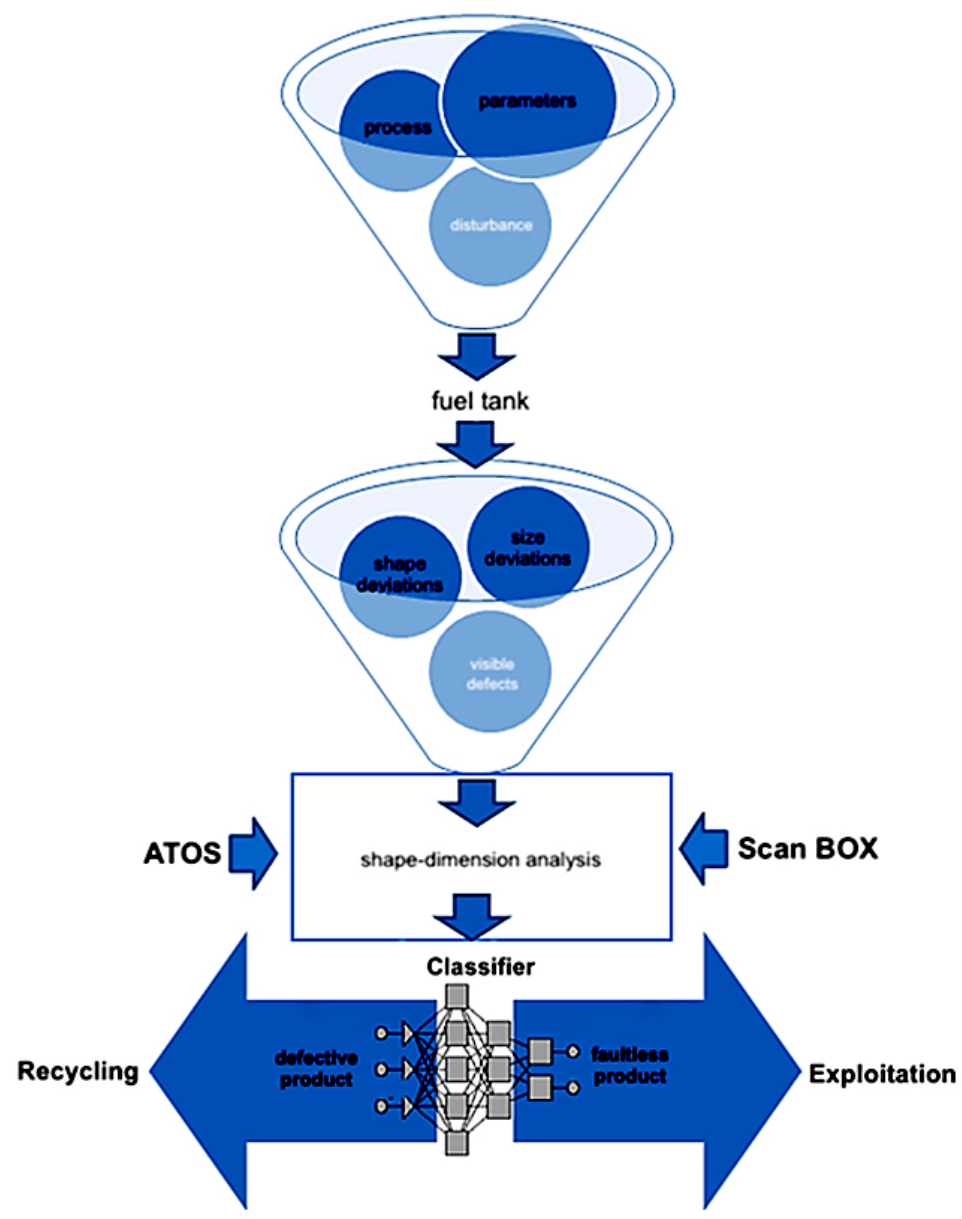

Fig. 11. Application of the Atos system in the structure of an intelligent classifier of the accuracy of manufacturing fuel tanks

mean value of the measuring deviations ranged from $-1 \mathrm{~mm}$ to $+1 \mathrm{~mm}$. Considering the dimensions of the tank and its multi-layer structure, these values are acceptable (excluding identified maximum deviation values) and constitute the basis for qualifying the tank as defective. This is indicated by high values of the decviations measures at selected measurement points. The mean standard deviations presented in the paper shows that with the increase in the value of measurement deviations, the values of the standard deviation also increase. The presented characteristic showed 


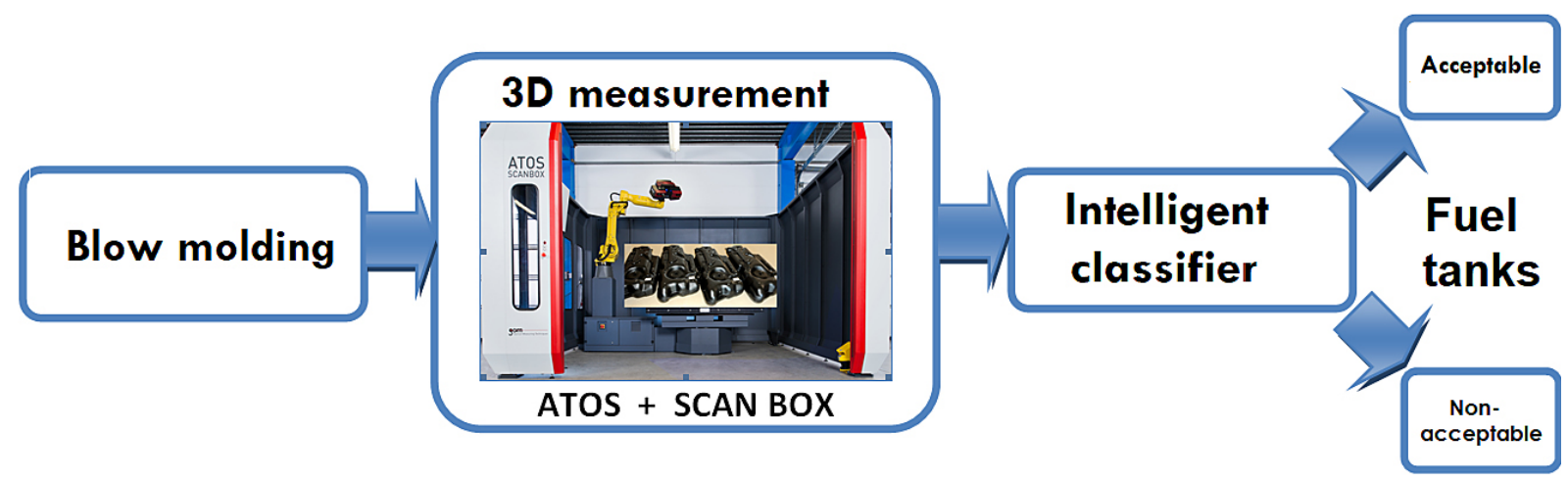

Fig. 12. The use of the Atos and Scan-box systems in the production line for the production of polymer fuel tanks

that they can be described by a linear function. The reasons for this are the effects of the processing process, such as processing shrinkage, multilayer structure and deformations resulting from thermal changes and variable wall thickness, as well as process instability. The mean values of the standard deviations are presented for a representative deviation of the $\Delta \mathrm{X}$ dimension of 0.1 ; 5; $10 ; 15$ and $20 \mathrm{~mm}$ were respectively: 0.014 ; $0.150 ; 0.172 ; 0.289 ; 0.340 \mathrm{~mm}$. This model can be used at a later stage during numerical analyses taking into account the changes in geometry obtained in the manufacturing process, i.e. taking into account all imperfections in the geometry of the analysed tank. In the modelling process, various types of details were abandoned, the importance of which is of secondary importance for the model intended for numerical and experimental research. Finally, it should be noted that the measurements of the geometry of passenger cars' tanks can be classified as pioneering. Due to the speed of measurements, they can be used to assess the phenomena accompanying the production processes by extrusion blow moulding, such as processing shrinkage, flash formation, etc. The measurement results can be used in the process of improving the quality of production of polymer tanks, optimization of their design and quick non-contact measurements. In addition, the specialized research equipment used in conjunction with the introduction of robotic stations (robot, rotary tables, linear tables, etc.) along with the presented methodology for measuring and processing the results may constitute the basis for the automation of the production line for the production of fuel tanks. In addition, the use of optical measurements using the ATOS system can be used to collect geometric information in a database that can be used to build an intelligent classifier of the quality of tanks manufactured in the production line. The methodology proposed in the work is the basis for the acquisition and analysis of measurement data for the creation of training data sets, testing and validation of intelligent neural models of the "black box" type of the intelligent classifier of dimensional and shape accuracy of multilayer polymer fuel tanks. The proposed model of the intelligent classifier will allow for active 3D recognition of correct and incorrect tanks. The advanced Scan-Box indicated in the paper, together with the Atos scanning system is an ideal solution in the production line of fuel tanks. Moreover, the material presented in the paper is an example of an innovative approach and may constitute an example (proposal) of a real application in an industrial plant producing polymer fuel tanks.

„The project/research was financed in the framework of the project Lublin University of Technology - Regional Excellence Initiative, funded by the Polish Ministry of Science and Higher Education (contract no. 030/RID/2018/19)".

\section{REFERENCES}

1. Józwik J., Dziedzic K., Barszcz M., Pashechko M. Analysis and Comparative Assessment of Basic Tribological Properties of Selected Polymer Composites. Materials. 2020;13:1-24.

2. Chen B., Wang J., Yan F. Friction and Wear Behaviors of Several Polymers Sliding Against GCr15 and 316 Steel Under the Lubrication of Sea Water. Tribology Letters. 2011;42:17-25.

3. Borba N., Blaga L., Dos Santos J., Amancio-Filho S. Direct-Friction Riveting of polymer composite laminates for aircraft applications. Materials Letters. 2018;215:31-34.

4. Pashechko M., Dziedzic K., Jozwik J. Analysis of Wear Resistance of Borided Steel C45. Materials. 2020;13(23):5529. 
5. Domagała I., Przystupa K., Firlej M., Pieniak D., Niewczas A., Biedziak B. Bending Behaviour of Polymeric Materials Used on Biomechanics Orthodontic Appliances. Materials. 2020;13(23):5579.

6. Li A., Zhang J., Zhang F., Li L., Zhu S., Yang, Y. Effects of fiber and matrix properties on the compression strength of carbon fiber reinforced polymer composites. New Carbon Materials. 2020;35;752-761.

7. Friedrich K. Polymer composites for tribological applications. Advanced Industrial and Engineering Polymer Research. 2018;1:3-39.

8. Kurdi A., Chang L. Recent Advances in High Performance Polymers-Tribological Aspects. Lubricants. 2019;7:2.

9. Farfan-Cabrera L.I., Tapia-Gaspar M., PérezGonzález, J. Tribology of Polymer Matrix Composites Within the Automotive Industry, Materials Science and Materials Engineering. Elsevier. 2021.

10. Sansoni G., Trebeschi M., Docchio F. State-ofthe-art and applications of 3D imaging sensors in industry, cultural heritage, medicine, and criminal investigation. Sensors. 2009;9:568-601.

11. Ruszniak P., Józwik J., Dziedzic K., Ostrowski D. Shearing strength test of orthopaedic titanium alloy screw produced in the process of $3 \mathrm{D}$ printing technology. Advances in Science and Technology Research Journal. 2017;11:128-137.

12. Lazarević D., Nedić B., Jović S., Šarkoćević Ž., Blagojević M. Optical inspection of cutting parts by $3 \mathrm{D}$ scanning. Physica A: Statistical Mechanics and its Applications. 2019;531:121583.

13. Park S.C., Chang M. Reverse engineering with a structured light system. Computers \& Industrial Engineering. 2009;57(4):1377-1384.

14. Wiesche S. Industrial thermoforming simulation of automotive fuel tanks. Applied Thermal Engineering. 2004;24:2391-2409.

15. Knyva V., Knyva M. Influence of 3D scanning data scattering to volume measurement of horizontal fuel tanks. Elektronika ir Elektrotechika. 2014;20(5):72-75.

16. Jozwik J., Piesko P., Krajewski G. Evaluation of QC10 Ballbar diagnostics method for CNC machine. Eksploatacja i Niezawodnosc - Maintenance and Reliability. 2010;3:10-20.
17. Józwik J., Ostrowski D., Milczarczyk R., Krolczyk G.M. Analysis of relation between the 3D printer laser beam power and the surface morphology properties in Ti-6Al-4V titanium alloy parts. Journal Of The Brazilian Society Of Mechanical Sciences And Engineering. 2018;40:1-10.

18. Parry E.J., Best J.M., Banks C.E. Three-dimensional (3D) scanning and additive manufacturing (AM) allows the fabrication of customized crutch grips. Materials Today Communications. 2020;25:101225.

19. Xu J., Xi N., Zhang C., Shi Q., Gregory J. Realtime 3D shape inspection system of automotive parts based on structured light pattern. Optics \& Laser Technology. 2011;43:1-8.

20. Semotiuk L., Józwik J., Kuric I. Measurement uncertainty analysis of different CNC machine tools measurement systems. Advances in Science and Technology Research Journal. 2013;7(19):41-47.

21. Börold A., Teucke M., Rust J., Freitag M., Recognition of car parts in automotive supply chains by combining synthetically generated training data with classical and deep learning based image processing. Procedia CIRP. 53rd CIRP Conference on Manufacturing Systems. 2020;93:377-382.

22. Hawryluk M., Ziemba J. Application of the 3D reverse scanning method in the analysis of tool wear and forging defects. Measurement. 2018;128:204-213.

23. Galanulis K., Reich C., Thesing J., Winter D. Optical Digitizing by ATOS for Press Parts and Tools. GOM. 2005.

24. Bere P., Dudescu M., Neamțu C., Cocian C. Design, Manufacturing and Test of CFRP Front Hood Concepts for a Light-Weight Vehicle. Polymers. 2021;13(9):1374.

25. Bere P., Neamtu C., Udroiu R. Novel Method for the Manufacture of Complex CFRP Parts Using FDM-based Molds. Polymers. 2020;12:2220.

26. Bere P., Neamtu C. Methodology for evaluate the form deviations for formula one nose car. Central European Journal of Engineering. 2014;4(2):148-154.

27. Neamțu C., Bere P. Methods for Checking the Symmetry of the Formula One Car Nose. Appl. Mech. Mater. 2014;657:785-789. 